\title{
A Testbed-based Approach to Develop Layer 3 Wireless Mesh Network Protocols
}

\author{
Kenichi Mase \\ Graduate School of Science \\ and Technology, Niigata \\ University \\ 8050, Ikarashi-2, Nishi-ku \\ Niigata city, Niigata, Japan \\ mase@ie.niigata-u.ac.jp
}

\author{
Yasunori Owada \\ Research Center for Natural \\ Hazard and Disaster \\ Recovery, Niigata University \\ 8050, Ikarashi-2, Nishi-ku \\ Niigata city, Niigata, Japan \\ owada@ie.niigata-u.ac.jp \\ Tomoaki Imai \\ Graduate School of Science \\ and Technology, Niigata \\ University \\ 8050, Ikarashi-2, Nishi-ku \\ Niigata city, Niigata, Japan \\ timai@net.ie.niigata- \\ u.ac.jp
}

\author{
Hiraku Okada \\ Center for Transdisciolinary \\ Research, Niigata University \\ 8050, Ikarashi-2, Nishi-ku \\ Niigata city, Niigata, Japan \\ hiraku@ie.niigata-u.ac.jp
}

\begin{abstract}
In this paper, two essential components of the layer $3 \mathrm{WMN}$, routing and rate-switch control, and initial network formation are discussed. The major part of routing protocol is composed of MANET (Mobile Ad Hoc Network) routing protocol and we have adopted OLSRv2, that is in progress of standardization in IETF MANET WG. Outdoor and indoor WMN testbeds are described to develop these WMN protocols together with some experimental results. A newly developed node prototype for WMNs and a WMN testbed built in rural hill-village areas are described together with some preliminary experimental results.
\end{abstract}

Categories and Subject Descriptors: C.2.2 [ComputerCommunication Networks]: Network Protocols - Routing protocols

General Terms: Experimentation

Keywords: Ad hoc network, OLSRv2, implementation, QualNet, testbed, mesh network.

\section{INTRODUCTION}

Wireless Mesh Network (WMN) is an emerging network concept and technology, aiming to remove wiring that is usually used to connect access points (APs) in conventional Wireless LAN (WLAN) [1]- [4]. Typically, each node of WMN has two or more wireless interfaces, one of them is used to accommodate legacy stations and others are used

Permission to make digital or hard copies of all or part of this work for personal or classroom use is granted without fee provided that copies are not made or distributed for profit or commercial advantage and that copies bear this notice and the full citation on the first page. To copy otherwise, to republish, to post on servers or to redistribute to lists, requires prior specific permission and/or a fee.

TRIDENTCOM 2008, 17th- 20th Mar 2008, Innsbruck, Austria. Copyright $\odot$ 2011- 2012 ICST ISBN 978-963-9799-24-0

DOI 10.4108/icst.tridentcom.2008.3144 to form WMN backbone. Since no wiring is required in principle, the cost and time to build WMN backbone is significantly reduced compared with conventional WLAN.

Several commercial products for WMN are already available in the market. Each product uses proprietary protocols and does not interoperate with each other. Standardization of WMN routing protocol is essential to promote wide deployment of WMN. In WMN backbone, when two nodes are within transmission range, they communicate directly over one-hop wireless link. Otherwise, two nodes communicate over multi-hop wireless path using other nodes as relay points. In this sense, the backbone of $\mathrm{WMN}$ is similar to the mobile ad hoc network (MANET) with the feature of multihop wireless communication although nodes in WMN may be stationary in most cases. Routing protocols developed for MANET may thus also be applicable to WMN backbone. Actually, MANET routing protocols such as AODV [5] and OLSR [6] have been used in many WMN testbeds.

WMN routing protocol may be realized in layer 2 or layer 3. The standardization of the layer 2 approach is in progress in IEEE 802.11s targeting relatively small-scale WMN [7]. We believe that layer 3 approach is also promising to extend the applicability of WMN. In this case, a MANET routing protocol being standardized in IETF MANET may also be used for WMN backbone.

In this paper, we describe our testbeds for layer $3 \mathrm{WMN}$ protocol development, presenting two essential components of the layer $3 \mathrm{WMN}$. Specifically, we discuss routing and rate-switch control, and initial network formation for WMN. We attach great importance to use standardized MANET routing protocol for the major part of WMN routing protocol, to assure interoperability and have adopted OLSRv2 [8], that is in progress of standard track RFC in IETF MANET WG. A kind of initial network formation protocol has been discussed in IEEE $802.11 \mathrm{~s}$, but we propose to realize this functionality at layer 3 using conventional IEEE $802.11 \mathrm{a} / \mathrm{b} / \mathrm{g}$ WLAN functionalities. 
Many testbed -based research and development on MWNs have been reported [2]- [4]. Our approach and contributions are caracterized by

- adopting a proactive MANET routing protocol with extended control message intervals.

- adopting OLSRv2 in progress toward standard track RFC to assure interoperability.

- configuring optimum rate for each link, termed "rateswitching".

- developing a series of testbeds, such as indoor, outdoor, campus and rural-hill area testbeds.

- providing real service to monitors.

- developing WMN initialization protocol boot.

The rest of the paper is composed as follows: Section 2 presents routing and rate-switch control. A large-scale outdoor WMN testbed and some experimental results are described. Section 3 presents initial network formation protocol. An indoor WMN testbed and some experimental results are described. Section 4 presents a testbed built in inhabitant area of rural hill-village. A newly developed node is described together with some preliminary experimental results. Section 5 concludes the paper.

\section{ROUTING AND RATE-SWITCH CON- TROL}

\subsection{Routing protocol}

\subsubsection{OLSRv2}

In layer $3 \mathrm{WMN}$, routing and forwarding are performed based on IP address. As mentioned in the introduction, it is natural to use routing protocols, that have been developed for MANET, also for WMN backbones. IETF MANET WG has been working to develop standard track RFC, one for each of reactive routing protocol and proactive routing protocol. They are DYMO [9] and OLSRv2, respectively. OLSRv2 is a successor of OLSR and inherits the major features and mechanisms of OLSR. Neighbor discovery functionalities using Hello message of OLSR is removed from OLSRv2 and is specified as an independent protocol, called Neighborhood Discovery Protocol (NHDP) [10]. There is a consensus that all MANET protocols use a common packet and message format (packetbb) and its standardization has also been in progress [11]. In this background, we choose OLSRv2 + NHDP as the routing protocol for WMN backbones. One reason to choose OLSRv2 is that it is a proactive routing protocol. In large-scale WMN, each node may accommodate many user terminals (mobile stations) with a significant amount of ingress and egress traffic. Multiple nodes may work as the gateways with outside networks such as the Internet. Taking these features into consideration, proactive routing protocols are expected to be more efficient than reactive routing protocols. One criticism for proactive routing protocols to be used for WMN backbones is that their control overhead is relatively high. It is possible, however, to reduce the control overhead by mean of longer periods for control messages, such as Hello and TC (Topology Control) messages in case of OLSRv2, assuming that most backbone nodes are stationary.

We have implemented the OLSRv2 routing protocol based on $[8],[10],[11]$, and name this implementation nOLSRv2 [12]. nOLSRv2 supports most functions of these specifications including attached network handling, multiple message aggregation to one packet, in-service adjustment of OLSRv2 parameters, multiple interface handling, IPv4 and IPv6, address compression. nOLSRv2 also supports the QualNet simulator [13].

\subsubsection{Supporting compatibility for simulator and routing daemon}

In designing nOLSRv2, Linux was chosen as the development platform for the real-time routing daemon, and the QualNet simulator as the simulation platform. In the second step, we divided the OLSRv2 functions into three parts; the Linux socket I/O part, the Qualnet I/O part, and the common OLSRv2 part. Removing platform dependent functions such as Linux and QualNet specific functions from the OLSRv2 source code, OLSRv2 common functions can be used both for the simulator, Linux and other platforms. This modular design makes it easy to port nOLSRv2 source code to different platforms.

\subsubsection{Address compression mechanism}

In generalized MANET packet/message format [11], compressed IP address representation is defined. Address compression algorithm is, however, not given in the specification and up to implementation. Address compression can be performed when some addresses used in MANETs have a common part. For example, multiple nodes of a MANET may have addresses with the same network prefix. When a node has multiple interfaces or multiple IP addresses with different network prefix at the same interface, the corresponding IP addresses may include the same host addresses. To compress a number of IP addresses into the corresponding compressed address representation (address block), it is necessary to extract the common parts from the IP addresses. Specifically, IP address is divided into two or three parts. When IP address is divided into three parts, each part is called "head", "mid" and "tail", respectively. In this case, head and tail appears only once and multiple mids are listed in the address block. Current nOLSRv2 does not have a mid part. In this case, an address block only has one head and multiple tails. The IP address compression algorithm for nOLSRv2 is as follows.

- Addresses are divided into some groups in terms of the first longest bytes match.

- For each group, an address block is created with one head and multiple tails.

\subsection{Rate-switch control}

The wireless LAN standards such as IEEE802.11a/b/g support multi-rate data transmission. In the auto-rate scheme, the optimum rate is dynamically searched and configured between the sender and receiver [14]. The auto-rate scheme is useful in conventional WLAN environment, where distance between a mobile station and an access point cannot be pre-determined and may change over time due to station mobility. However, it is not always effective in a WMN 


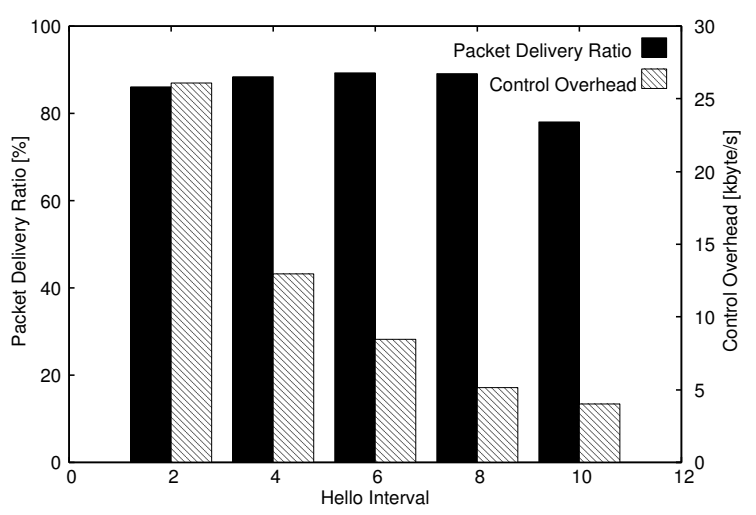

Figure 2: Relationship between control overhead, packet delivery ratio and Hello interval.

\subsection{Overview}

We consider building a WMN in a given area. We assume that locations of nodes are pre-selected. Nodes are placed at the respective locations and powered. They are then expected to perform initialization functionalities such as automatically discovering their neighbor nodes, determining a common channel, and establishing links with the neighbor nodes to form a WMN. We call this initialization process initial network formation. The functionalities to realize the initial network formation in layer 2 have been discussed in IEEE 802.11s. We seek to develop initial network formation protocol for layer $3 \mathrm{WMN}$, assuming conventional IEEE $802.11 \mathrm{a} / \mathrm{b} / \mathrm{g}$ WLAN functionalities.

\subsection{Protocol description}

We assume that each node has two wireless interfaces and one interface is to be set to a common channel to form WMN backbone during initial network formation. Other interface may be used as that of access point to associate with legacy mobile stations. When nodes have more than two wireless interfaces, multiple interfaces may be used to form WMN backbone with an appropriate channel assignment (target network). Forming initial network is the first step to form the target network, that is beyond the scope of this paper. In the following, we focus on the formation of the initial network. We also assume that all nodes have preset WMN parameters including the value of SSID to start initial network formation. The proposed protocol is composed of parent and child functionalities. Initially, one predetermined node (initial-parent node) works as the parent and others do as the child. Manual configuration may be used to select the initial-parent node. Otherwise initial-parent-selection protocol may be developed and used. Initial-parent-selection protocol is beyond the scope of this paper.

Parent node sets one of its wireless interfaces as communication interface, sets SSID to that of the preset WMN parameters and activates it with ad hoc mode. At this moment, parent node is free to choose an appropriate channel and BSSID for the communication interface. Other nodes (child-nodes) set two of its wireless interfaces to searching interface and communication interface, respectively, and activate the searching interface with managed mode. Child nodes start to search any parent node using the searching interface through channel scan. When a child node discov- ers a node running as the parent node with ad hoc mode, it obtains the WMN parameters of the parent node. If they coincide with those of its own, it judges that the discovered node is a legitimate neighbor node of the WMN under formation. It sets the corresponding SSID, channel and BSSID, used by the parent node, to the communication interface and activates it with ad hoc mode. It may deactivate the searching interface. At this moment, this node also starts to work as the parent node. In this way, the number of nodes operating as the parent node gradually increases and eventually reaches the stable state, where all nodes operate as the parent node with the same SSID, channel and BSSID at their communication interfaces.

\subsection{Testbed}

The 12 node indoor testbed was built in three floors of a building of Niigata University campus in 2006. The node's location is shown in Fig. 3. A node is composed of a compact computer (same as described in 2.3 ) and IEEE 802.11a/b/g wireless LAN card (NEC AtermWL54AG with Atheros chipset, Madwifi driver) inserted for communication interface and IEEE 802.11b wireless LAN card (BUFFALO WLI2-CF-S11 with HERMES chipset, and PLANEX GWNS11H with PRISM chipset, HostAp driver) for searching interface.

\subsection{Experiments and results}

In this experiment, one of the nodes is pre-selected as the initial-parent node and others are set as the child nodes. All nodes start to work simultaneously. Each node runs routing protocol at the activated communication interface. We use an implementation of OLSR [17]. (nOLSRv2 described in 2.1.1 was not matured at this time). Each node other than the initial-parent node measures the time to establish the common channel for the initial network (Initial network joining time). The initial network joining time for the last node is termed as the initial network formation time. The initial-parent node can monitor the number of nodes joined in the WMN backbone based on the information obtained from the routing daemon (OLSR) and measures the time for all nodes to work as the member of the WMN (the initial network working time). We select every node as the initial-parent node in turn and repeat 20 experiments for each case. Channel 10 is selected at each initial-parent node in all experiments. Maximum, minimum and average time is obtained for each of initial network joining time, initial network formation time and initial network working time, respectively.

Initial network formation and working times for different

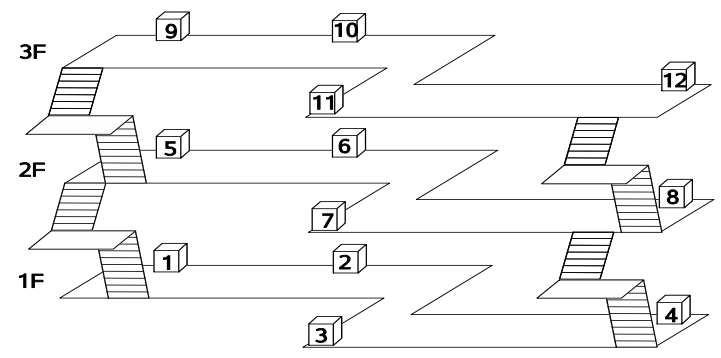

Figure 3: Indoor WMN testbed node placement. 


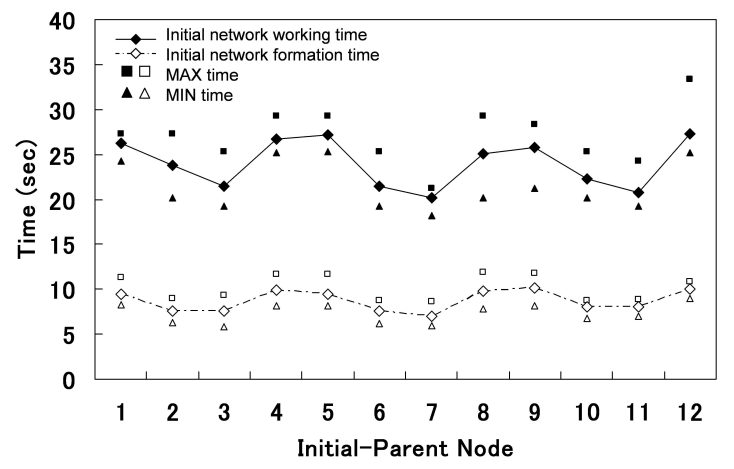

Figure 4: Initial netwrok formation time and working time.

initial-parent node are shown in Fig. 4. When node 1, 4, 5, $8,9,12$ is selected as the initial-parent node, initial network formation time is relatively longer. This is because these nodes are located at the edge of each floor and more hops are required to reach far-end nodes. On the other hand, when nodes located around the center of the testbed, ex. node 7 , are selected as the initial-parent node, initial network formation time is minimized.

Initial network working time for different initial-parent node has almost similar trend as that of initial network formation time. The difference between initial network formation time and initial network working time is around 15 seconds, that is needed for OLSR to establish routing table at each node.

\section{RURAL HILL-VILLAGE AREA TESTBED}

\subsection{Background}

Yamakoshi is a small village, located in a rural-hill area, and about $20 \mathrm{~km}$ far from the nearest town. No commercial broadband service is available at present there because of its geographical conditions. Yamakoshi-net joint experiment project started in May 2006 is a five year project and is led by Niigata University with support and collaboration of NTT east group, KDDI, Shinetsu Bureau of Telecommunication, Niigata Prefecture and Nagaoka City. The aims of the project is to build a WMN testbed, called Yamakoshinet, in Yamakoshi Village and to perform experiments using this testbed to study networking technologies for deploying economical and disaster-tolerant communication networks for the rural hill-village areas.

\subsection{Network Structure}

Overview of the Yamakoshi-net is shown in Fig. 5 [18]. Yamakoshi village consists of more than ten small settlements. Two settlements, Takezawa and Mushigame are covered by the WiFi-based layer $3 \mathrm{WMN}$ using 8 and 12 pole top antennas and nodes, respectively. The two settlements are each located about 1 mile from the local-government office, and connected to the office with the broadband wireless access systems using $5 \mathrm{GHz}$ band, respectively. The localgovernment office is directly connected to Niigata University with the $100 \mathrm{Mbps}$ commercial wide-area Ethernet service.

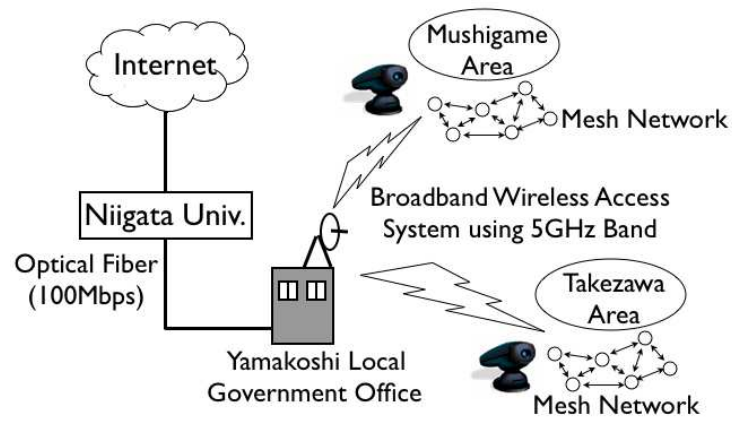

Figure 5: Yamakoshi-net overview.

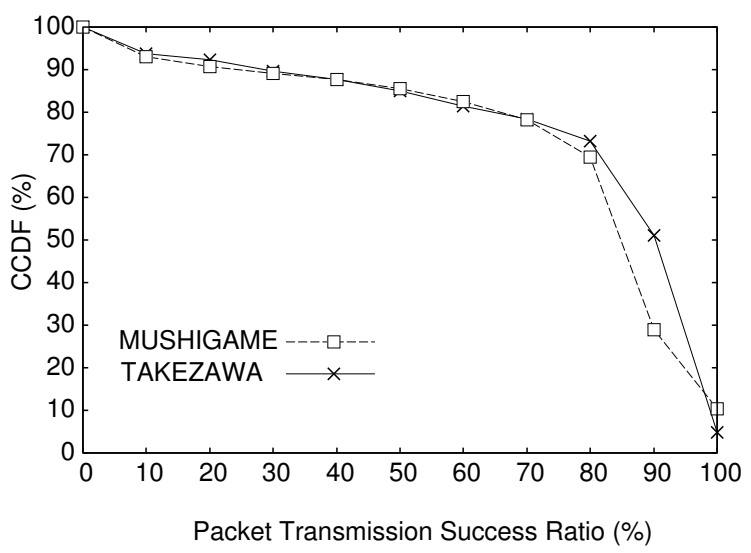

Figure 6: The complementary cumulative distribution function $C C D F$ of the packet transmission success ratio of the links.

All experiments can be performed remotely from Niigata University.

\subsection{Node structure}

The node consists of a compact computer (same as described in 2.3), two IEEE $802.11 \mathrm{a} / \mathrm{b} / \mathrm{g}$ wireless $\mathrm{LAN}$ card (NEC AtermWL54AG with Atheros chipset, Madwifii driver) and an IEEE $802.11 \mathrm{~b} / \mathrm{g}$ wireless LAN access point (Corega CG-WLAPGEX with Atheros chipset). The two wireless cards of the compact computer are operating in an ad hoc demo mode using Madwifi wireless drivers. They are used for different purpose. One is used to control the nodes from the control station at Niigata University with channel $\# 1$, and termed control interface. Another is used to transmit data packets with channel \#5, and termed communication interface. The wireless LAN access point to provide a wireless LAN spot service is connected to the computer's Ethernet interface. Two wireless cards and one access point are connected to three whip antennas attached to the outerside of the node box, respectively. One of them is attached on the upper side of the node box and the others are on the lower side. The antenna is $5 \mathrm{dBi}$ gain, non-directional in the horizontal, and directional in the vertical direction between +10 and -5 degree.

Temperature sensor is installed in the node box. When the temperature in the box becomes higher than the threshold, a fan is automatically activated to regulate temperature. The 
power of the node is reset twice a week in order to avoid the loss of control by operation mistakes.

Yamakoshi-net provides service to residences in Takezawa and Mushigame settlements. At each residence an access point, same as used for the nodes of the backbone, operating with the managed mode, is used to associate with the access point of the nearby backbone node. Each backbone node has its own subnet address, that is advertised as the attached network set to other nodes by means of the OLSRv2 TC message. User computer is connected to the residence access point with the Eathernet interface and obtains an IP address from the DHCP server residing at the corresponding backbone node.

\subsection{Results of preliminary experiments}

We have conducted the preliminary experiments to evaluate basic connectivity, that is, the packet transmission success ratio of the links of the Yamakoshi-net. The measurement is executed 4 times per day (at 0,6,12, and 18) for 3 days, thus the number of total measurements is 12 . At each time, every node broadcasts packets by turns, and the other nodes receive and recode the received packets. The size of broadcast packet is 1472 bytes, the packet transmission rate is four packets/sec, and the total number of transmitted packets is 1000 packets. The measurement result is shown in Fig. 6. This figure shows the complementary cumulative distribution function (CCDF) of the packet transmission success ratio of the links. The packet transmission success ratio is $80 \%$ or more for $70 \%$ links, revealing enough network connectivity.

\subsection{On-going service evaluation by monitors}

During the winter in 2006, some of the antennas did not work because snow was attached and frozen on the spiral part of some antenna poles. we replaced the antennas with straight pole antennas. Since then Yamakoshi-net has been operating well even during the cold winter as well as hot mid-summer.

We have invited applications for monitors of the Yamakoshi-net in August 2007. As the results, we have successfully obtained 9 monitors. We are currently providing teleconference and Internet access services to monitors beginning from October 2007. In this trial, we will use nOLSRv2 described in 2.1.1 as the routing protocol for WMN backbone and will investigate the optimum values of parameters including Hello and TC message intervals, and the benefits of rate-switch control. We will also conduct survey on user satisfaction with regard to service and communication quality.

\section{CONCLUSIONS}

In this paper, we described a testbed-based approach to develop layer $3 \mathrm{WMN}$ protocols. Specifically, two essential components of the layer $3 \mathrm{WMN}$, layer $3 \mathrm{WMN}$ routing, rate-switch control and initial network formation, were identified and discussed. As the major part of WMN routing protocol, we have adopted and implemented OLSRv2, that is in progress of standardization in IETF MANET WG. Outdoor and indoor WMN testbeds in Niigata University campus were described to develop these WMN protocols together with some experimental results. A newly developed node prototype for WMNs and a WMN testbed built in Yamakoshi, Nagaoka city, Yamakoshi-net, were described together with some preliminary experimental results and ongoing monitor evaluation.

Standardization of OLSRv2 including related protocols (NHDP, etc.) and packet and message format (packetbb) are not yet completed. We will continue to develop implementation of OLSRv2 together with related protocols to reflect the latest standardization using our testbeds. We will also develop full functionalities of WMN protocols and verify performance using the testbeds as well as continue to provide service to monitors. We expect to obtain useful feedbacks from monitors of Yamakoshi-net with regard to the benefits and issues of WMN-based broadband Internet access in rural hill-village areas.

\section{REFERENCES}

[1] I. F. Akyildiz, et. al., "Wireless Mesh Networks: A Survey," Computer networks, Vol. 47, No. 44, pp. 445-487, Elsevier Science, 2005.

[2] http://pdos.csail.mit.edu/grid/

[3] J. Bicket, et. al., "Architecture and Evaluation of an Unplanned 802.11b Mesh Network," MobiCom'05, Sept. 2005.

[4] R. Draves, et. al., "Routing in Multi-Radio, Multi-Hop Wireless Mesh Networks," MobiCom'04, Sept. 2004.

[5] C. Perkins, et. al., "Ad hoc On-Demand Distance Vector (AODV) Routing," RFC 3561, July 2003.

[6] T. Clausen et. al., "Optimized Link State Routing Protocol (OLSR)," RFC 3626, Oct. 2003.

[7] IEEE P802.11s/D1.02, March 2007.

[8] T. Clausen, et. al., "The Optimized Link-State Routing Protocol version 2," draft-ietf-manet-olsrv2-03, Work in Progress, Feb. 2007.

[9] I. Chakeres, et. al., "Dynamic MANET On-demand (DYMO) Routing," draft-ietf-manet-dymo-08, Mar. 2 2007.

[10] T. Clausen, et. al., "MANET Neighborhood Discovery Protocol," draft-ietf-manet-ohdp-02, Feb. 2007.

[11] T. Clausen, et. al., "Generalized MANET Packet/Message Format," draft-ietf-manet-packetbb-04, Jan. 2007.

[12] Y. Owada, et. al., "OLSRv2 Implementation and Performance Evaluation with Link Layer Feedback," IWCMC'07, Aug. 2007.

[13] http://www.scalablenetwork.com/

[14] A. Kamerman, et. al., "WaveLAN II: A high-performance wireless LAN for the unlicenced band," Bell Labs Technical Journal, pp. 118-133, 1997.

[15] S. Obana, et. al., "Test-Bed Based Research on Ad Hoc Networks in Japan," IEICE TRANS. COMMUN., VOL.E88-B, NO.9, 2005.

[16] T. Shirogane, Niigata Univ. Master Thesis, 2008.

[17] http: //www.reseaucitoyen.be/wiki/index.php/Unik0lsr

[18] Y. Takahashi, et. al., "A Wireless Mesh Network Testbed in Rural Mountain Area," WiNTECH'07, Sept. 2007. 